\title{
Increasing the quality and enhancing musical auditory notions through professional training in piano
}

\author{
Krasimira Georgieva Fileva-Ruseva \\ Faculty of Music Pedagogy, Academy of Music, Dance and Fine Arts - Plovdiv, Bulgaria, Europe \\ Email address: \\ krassyfilleva@abv.bg
}

To cite this article:

Krasimira Georgieva Fileva-Ruseva. Increasing the Quality and Enhancing Musical Auditory Notions through Professional Training in Piano. International Journal of Literature and Arts. Special Issue: Musical Theory, Psychology and Pedagogy.

Vol. 2, No. 5-1, 2014, pp. 25-32. doi: 10.11648/j.ijla.s.2014020501.15

\begin{abstract}
One of the areas of art, where specific abilities are combined and their maximal and balanced development is required, is the activity of piano performance. For this reason, at the first place among the responsible tasks of the piano pedagogue (who teaches the future interpreters) is the development of the musical abilities of the student - the mode sense, methro-rhythmic sense and musical auditory notions. Musical auditory notions allow for sound impressions of varied musical sequences to be stored in the individual's memory pool. The musical auditory experience, containing the gathered musical auditory notions, facilitates each tonal perception through identification and differentiation of the familiar, common (already stored in the memory), from the new, different, which is to be imprinted in the mind and on its turn used as a basis for future perception. The richer, more detailed, well comprehend and easily activated the musical auditory notions of the student, the more facilitated and of more quality will be the process of comprehension, learning and artistic performance of the tonal art. The professional training in piano is aimed mainly at improving the instrumental skills, development of a repertoire and building a style and general musical culture. Among the reasons for the insufficient attention of piano pedagogues on the balanced development of the musical abilities of their students, is the limited time frame of the piano lesson, as well as the requirements for the pedagogue to sent the largest possible number of prominent students to as many as possible competitions for pianists, auditions, youth festivals and concerts. The pianist being held continuously in their everyday activities on the same and only concert or competition program for reasons of assuring the maximal quality of its performance on a specific forum, leads to the creations of a relatively sparse pool of musical auditory notions. From the supplementary activities in piano training, related to the process of creation and actualization of musical auditory notions are mainly the following: reading at first glance, playing by ear, recreation from memory of works that have not been performed recently. More connected to the accumulation of a pool of musical auditory notions is the schematic learning; to the particularization and comprehension of the musical memory image, introduced in the pool of musical auditory notions through its multiple actualizations, is related the analysis of the musical work of art with the purpose of memorization and clarification of the interpreter's conception.
\end{abstract}

Keywords: Music, Musical Auditory Notions, Piano Training

\section{Introduction}

For the successful performance of any activities, the relevant abilities are required to be present. This is especially valid in arts, where without the necessary qualities of the person the creative act would be incomplete or would not be achieved at all. One of the areas in art, where the particular abilities are combined and their maximal and balanced development is required is the activity of the piano performance. For that reason, first place among the responsible tasks of the piano pedagogue (who is teaching the future interpreters) is the development of the main musical abilities of the student - mode sense, methro-rhythmic sense and musical auditory notions. The capability of the pupil to recreate faithfully a musical thought, shows that they have perceived it namely as a meaningful and logical musical sequence, with the emotional and semantic meaning it is carrying. In order for it to be comprehended in such a way, the musical abilities of the student have been activated - mode sense, through which the moments of intensification and of decreasing of the 
pressure in the melody are identified and realized; methro-rhythmic sense, with the help of which the pulse, temperament of the musical character is being comprehended; musical auditory notions, through which the perceived music is being compared with the already familiar, in order to find the style it belongs to, the national specifics, the musical symbolism, embedded in it.

\section{Subject, Aim and Tasks of the Study}

Musical auditory notions are an ability, which is often neglected in the piano methodology literature. This is not justified, since musical auditory notions, apart from the possibility of mentally "listening" to sheet music, include also the musical memory, with the help of which the essential activity of the pianist-interpreter is performed - the recreation of the tonal work before an audience. The musical auditory pool contains to a great degree also the musical style culture of the performer.

For this reason, the aim of the present study is, on the basis of knowledge of the essence of musical auditory notions, to present effective approaches of working for their enhancement and the improvement of their qualities.

The tasks, deriving from this aim, are to:

1. Unveil the characteristics of the musical auditory notions, which are susceptible to pedagogical influence.

2. Clarify the reasons of the insufficient attention of piano pedagogues on the enhancement of musical auditory notions.

3. Offer methods for increasing the quality and enhancing the musical auditory notions.

4. Present arguments for the efficiency of the proposed methods.

The subject, on which the present study is focused, is the musical auditory notions of the pianist-performer.

\section{The Essence of Musical Auditory Notions}

Musical auditory notions are a musical ability, which allows storing sound impressions from varied musical (melodic and methro-rhythmic) sequences in the memory pool. The musical auditory experience, built on the basis of musical auditory notions facilitates each tonal perception through identification and differentiation of the familiar, common (already stored in the memory), from the new, different, which is to be imprinted in the consciousness and on its turn will be used as a reference point for the next perception. The richer, more detailed, well comprehend and easily activated the musical auditory notions of the student, the more facilitated and of more quality will be the process of apprehension, learning and artistic performance of the tonal work.

The piano is a polyphonic musical instrument, which means that the musical auditory notions, acquired through working on clavier pieces, are significantly more elaborate and complex, compared to those, acquired when learning pieces for a monophonic instrument. This imposes also the greater requirements for the development of musical auditory notions in pianists, for this musical ability to be fully developed and used in the piano performer's practice.

\section{Characteristics of the Musical Auditory Notions}

Musical auditory notions are gathered during the process of musical education of the person, but apart from the wealth of the acquired musical notion pool (which, although cannot be measured in the separate individual, because there is no option of registering all the musical auditory notions, received through involuntary memorization, can be considered a quantitative characteristic), this ability has also qualitative features. Those are:

\subsection{Speed of Memorization}

Aside from the personal specifics of the one performing the mnemonic activity, the speed depends on how many and what types of memory are engaged in the memorization; on the conditions, in which the memorization itself is being performed; on the position of the object of the mnemonic activity (if the musical play is the goal of the memorization, it will be remembered in more detail than the music, which sounds as a background to another activity); on the motivation of the performer.

\subsection{The Reliability of Storing the Memorized Musical Works, Intonations, Rhythms, Assonances and their Logical Sequences; the Possibility of Lastingly and Efficiently Memorize the Learnt Piece}

Naturally, this characteristic (as well as the one discussed above) is related to personal traits, but also to the way of memorization, i.e. the circumstances, on which the speed of memorization depends, are also valid for it).

\subsection{The Level of Details of the Notion's Image}

The quality of the musical auditory notions varies also in terms of the detail of the memorization and the ability of the performer to mentally picture it with its particular features. The details allow us, for example, to mentally "playback" to a given melody with a specific timbre, in a strictly defined register, with marked articulation, even with the specific for a given musical instrument effects (for example, the usage of the right pedal of the piano). Regarding the polyphonic music, details help also recreate mentally the textural elements within the work with the articulation characteristics (marks, timbre nuances), with which we have perceived them. The same details allow us to "retell" - to play in our head the piece, which we are preparing for a concert performance, verifying the reliability and durability of the formed memory image. 


\subsection{Flexibility of Musical Auditory Notions}

Flexibility is related to the ease of evoking the memory image. It provides also the possibility to mentally change the characteristics of sounding of a specific musical element, to compare the characteristics and to select the most suitable ones, to picture the sound as a whole (even a few different variants of sound), to remember the especially valuable "finds".

\subsection{The Speed and Depth of Comprehension of the Meaning of the Music Heard}

The state of these two properties depends on the ability to opportunely compare what is being heard with the memory pool of templates; on the variety and quality of the stored memory images. The comprehension of the meaning of the musical work relates to unveiling its structure and emotional contents. This means that it is of extreme importance to clarify the musical auditory notions as regards to the form of the newly perceived piece, the dynamics of the feelings, which it incites, as well as the knowledge about the structure and emotional characteristics of the works, which comprise the musical auditory pool.

\section{Reasons for the Insufficient Work on Piano Pedagogues on Enhancing the Musical Auditory Notions}

The professional education in piano is focused mainly on learning the instrument playing skills, building up a repertoire and developing a stylistic and general musical culture. Thus, the development of the musical abilities of the student pianist usually remains the responsibility of the teacher in solfège. Among the reasons for the insufficient attention being paid by piano pedagogues on the balanced development of the musical abilities of their pupils, is the limited timeframe of the piano lesson, which does not leave enough time for supplementary activities, as well as the requirements for the pedagogue to present the greatest possible number of prominent students at as many as possible competitions for pianists, auditions, youth festivals and concerts. From this derives the need for a relatively small volume of, at the same time technically complex (in order for it to be presentable), material to be prepared for a long period of time and the type of music, which is being taught to be chosen according to the regulations of the competitions, the stylistic criteria of the festival or the concert and the presentability, "impressiveness" of the pieces. All of this limits the choice of material, imposes a certain degree of one-sidedness as regards to the author's way of expression and results in the student being familiar with a modest in volume and unvaried in style repertoire. Unfortunately, this approach is applied predominantly on the most gifted students. In view of the tremendous in volume clavier musical treasure that has been created and taking into account the fact that almost all composers have created works for the piano, i.e. this is the richest solo instrumental literature - such deprivation of the pianists should not be allowed. The limited repertoire creates unvaried in intonation and rhythm impressions, since each author has a specific language of rhythm and intonation, which comprises their individual creative way of expression.

The pianist being held continuously in their everyday activities on the same and only concert or competition program for reasons of assuring the maximal quality of its performance on a specific forum, leads to the creations of a relatively sparse pool of musical auditory notions, i.e. represses the development of the discussed musical ability. One of the measures to prevent this negative manifestation of the aspiration for perfection in instrument playing and to provide the suitable opportunities for performance of the student will be to find a way to include among the criteria for selecting educative material the one of the stylistic variety. This is feasible, for example, when deciding on pieces, which are featured in the regalements of the competitions as works by the choice of the candidate. The thoughtfully selected, and according to the requirements for stylistic variety, works of the free program, which are also different in style in each subsequent competitions, for which the young piano player is preparing, are one of the possible ways of providing conditions for the development of the musical auditory notions during piano classes.

\section{Activities, Related to the Enhancement of the Musical Auditory Notions}

The development of this musical ability in the education in piano is realized through three types of activities.

The first are directed towards activating the very process of creation and evocation of the musical auditory notions, i.e. the object of training is the capability of quickly, accurately and in detail actualizing the mental image of a piece, which is already familiar to the pianist, or the preliminary creation of a notion for music, which is to be heard (new, unknown at the moment).

The second type of activities address mainly the building of the most extended possible memory pool, of a reserve of set images, with which the newly perceived music will be compared and which is constantly enriched in the course of acquiring professional experience.

The third type of activities concerns the clarification, systematization of the memorized information, finding common traits, regularities.

All three of the discussed types of activities are related to the qualitative, as well as to the quantitative characteristic of the musical auditory notions. Practiced on a large in volume piano material, the activities, focused on the multiple activation of the process of building the musical auditory notions, will result in a quantitative overlay, but the experience, which is acquired when applying them on a vast piano work literature has a relation also to the improvement 
of the quality of the created notions, as for example the capability to easily evoke and recombine them in the mind (their flexibility). The constant enhancement of the memory pool, which is a quantitative characteristic, creates a more expanded base for comparison to the newly heard music. This is manifested, however, also in the higher quality of the memory image, since the enhanced base for comparison implies the presence of more realized regularities of logical sequences. When many and varied sound sequences (melodies and polyphonic works) are lastingly remembered, when the regularities, their shared traits are identified, discerned and arranged in the mind, working with their elements becomes faster and easier, it provides the ability to more quickly find and more deeply understand the specific in the newly perceived music.

Among the main activities in piano training, especially directly related to the musical auditory notions, is memorization by heart, done by the student, which includes also different approaches of verification of the memorized information; and repertoire policy of the pedagogue.

Learning by heart of the musical work, which, in order to be more detailed, long-lasting and reliable, has to be achieved consciously, is a process of forming a specified in detail, full and long-lasting musical auditory notion. This notion is added to the pool of musical auditory notions, gradually enriched with the building of the professional experience of the pianist. From this follows that memorization is connected in the first place to the creation of musical auditory notions and the enhancement of the notion pool. With frequent practice, memorization becomes more efficient, which means that this trains and improves the very process of creation and evocation of the musical auditory notions. Through memorization, which is conscious and achieved through the help of analysis of the form and texture, we create more clarified and systematized musical auditory notions; this in its turn helps their reliability and flexibility.

The different forms of verifying the quality of memorization consists in actualization, and therefore further consolidation of the specific musical auditory notion. When verifying the accuracy, completeness and durability of the musical auditory image, which is a necessary action, when we are dealing with a piece, being prepared for stage performance, it is very useful to mentally play the piece. This mental playing, though, not only gives a fuller picture of the state of the memorized image of the work (all the elements, which have been memorized incompletely or unconvincingly, become apparent), but it also helps improve the level of detail and the flexibility of the musical auditory notions, i.e. has direct relation to improving the quality of the process of creation and actualization of the musical auditory notions.

Repertoire work has to be oriented not only towards the selection of pieces, through which the instrumental skills will be developed and through which the student will be presented in the best light, but also towards the enhancement of the pool of notions of the student. In order to provide the needed variety of this pool of notions, it is useful to seek for opportunities to learn not only pieces, which are varied in style and texture (an invariable requirement), but also pieces, with a modal structure, different from the classical major and minor, i.e. where the relations between the degrees are not based on the principle of stability/instability. In order to clarify this, I will apply the following examples:

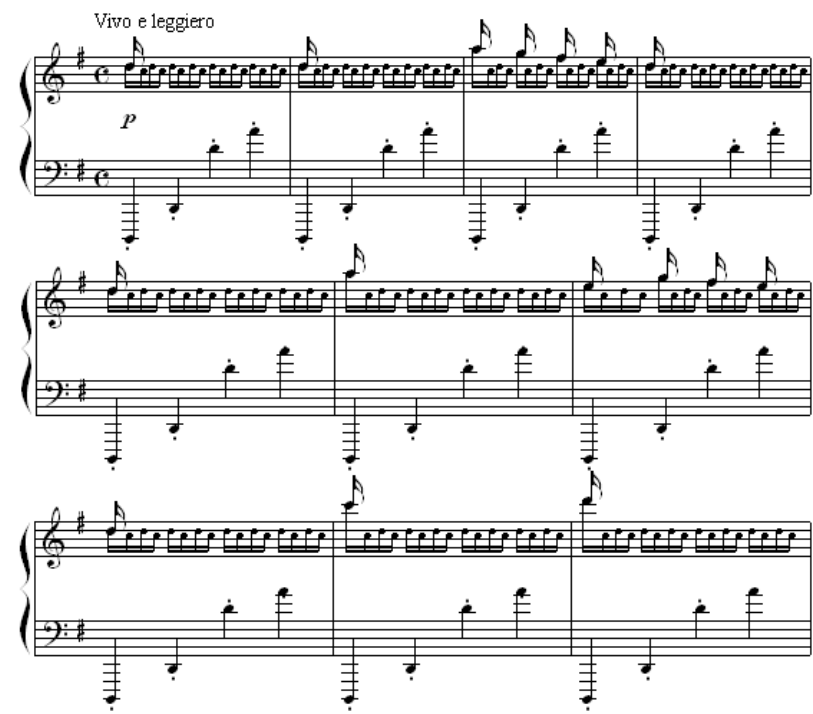

Figure 1. Dimitar Nenov "Gaida", b. 1-10 (mixolydian modal coloring)

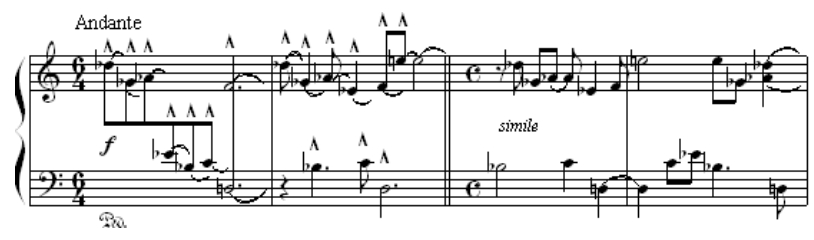

Figure 2. V. Kazandjiev "Kambanen Zvan" ("Sound of Bells"), b. 1 - 4 (artificial modal structure)

The beginning of the play is built over the artificial modal structure:

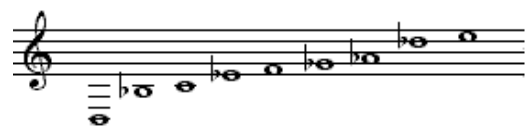

Figure 3. The artificial modal structure used in the first 9 bars of clavier miniature "Kambanen Zvan" ("Sound of Bells") by V. Kazandjiev

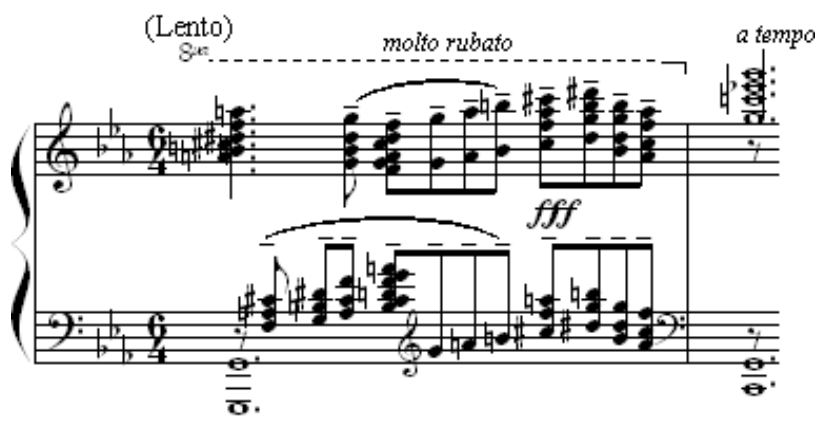

Figure 4. P. Vladigerov "Esenna Elegia" (Autumn Elegy), b. $30-31$ (the specific excerpt - the moment of culmination of the play - has a whole-tone sound line. 
Of course, the last excerpt is too short, but its brightness, unusual sound and its impact provide reason to use it as an example and to form a distinct musical auditory notion.

The diversification of the musical auditory notions pool is needed to be achieved also in relation to the methro-rhythmic organization. It is useful to seek musical pieces with a more complex combination of rhythmic groups (Figure 5), as well as plays with varied genre characteristics, since one of the signs, which we use to determine the genre of a certain play, is namely its methro-rhythmic organization - its characteristic meter, typical rhythmic groups, tempo. Interesting in regard to their methro-rhythmic organization are also the works with compound time signatures (Figure 6).

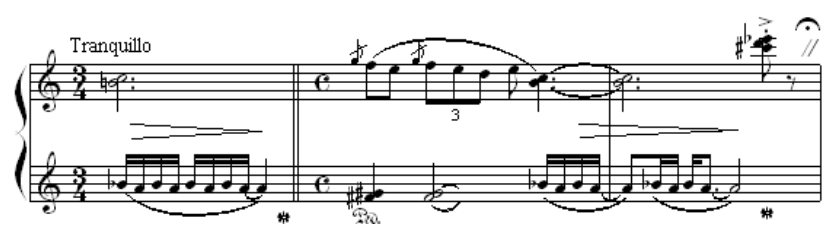

Figure 5. V. Kazandjiev "The Cat 'Purr', b. 3-8

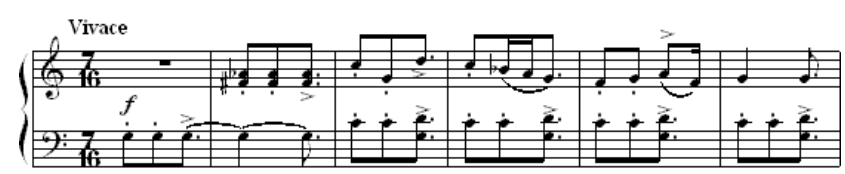

Figure 6. V. Kazandjiev "Rachenitza. Bulgarian Dance ", b. 1 -6

Apart from considering the repertoire, provisioned for the main work during the classes in piano, relevant to the improvement of the musical auditory notions is also the practice of the so called "supplementary activities" (term by A. Shtapov).

Mainly, relevant to the process of creation and actualization of the musical auditory notions are the supplementary activities like reading at first glance, playing by ear, recreating from memory pieces that have not been performed recently. More connected to the accumulation of a musical auditory notion pool is the schematic study, while the analysis of the musical work for memorization and for clarification of the interpreter's conception is related to specification and comprehension of the musical memory image, being introduced in the pool of musical auditory notions through its multiple actualizations. Of course, this differentiation is a conditional one; each activity, when it is correctly performed is valuable for the improvement of the quality of all three processes, characterizing the musical auditory notions.

Before performing a prima vista, the student goes over the sheet music, gets to know the key signature signs, the time signature, the markings for tempo and for changes in the tempo, mentally reads through some of the more complicated rhythmic groups, assonances, creates an imprint of the type of the texture. In order to train the ability of creating a musical auditory notion of the piece, before it has been played on the piano, it is appropriate to prompt for a detailed mental reading of the sheet music, without playing on the musical instrument. This way of creating a musical auditory notion, since during silent reading there is no actual music heard, is based on the re-combination of elements of notions, comprising the musical memory pool of the student. In this approach of forming the notion's image, greater flexibility of the musical auditory notions is achieved, which is a needed quality for the piano performer. Reading at first glance affects to a certain degree the enrichment of the musical auditory pool. It is true that the play, performed a prima vista, remains for a short period of time within the attention of the pianist, which at first glance does not lead to its memorization. The very reading, however, is realized at a great pressure, since it is performed in almost real time (one of the criteria for successful reading at first glance is the playing in a tempo, close to the actual one) and with the requirement for the best possible recreation of the character of the work; the greater pressure on the attention creates certain conditions for imprinting the playing of the piece (even though not in as much detail) in the memory of the instrumentalist.

In order to train clavier interpreters, who possess vivid and detailed musical auditory notions, accurate, extensive and lasting musical memory images, it has been suggested in Russian piano methodical literature to also practice the activity playing by ear even after the period of the initial acquaintance with the piano instrument; but now this should be done as a supplementary activity, concomitant with the main ones in the class. Since enough time for supplementary activities cannot be spared in every lesson, it is appropriate for the student to acknowledge the gain from playing by ear for the creation of musical auditory notions and flexible easily re-combinable visual-auditory-motion connections between the tones and their places on the keyboard, so they can perform this activity during their out-of-class training.

Memory retrieval of plays, which haven't been playing soon (A. Shtapov), less frequently used in the piano practice, is a valuable means of achieving an easy reproducibility (flexibility) and reliability of the musical auditory notions, i.e. it is closely related to the activation of the process of their creation and animation.

Specification of the musical auditory notions is achieved when the pedagogue explains to the student or, if the student has acquired theoretical knowledge - require from them to formulate conclusions for the specifics of the form of the work, modal and methro-rhythmic organization, the sound vertical and the logic of the chord sequences. Developing a habit of using the correct terms helps the practical applicability of the assimilated theoretical information, trains the necessity to analyze the work and provide argumentation for the performer's vision; facilitates the building of orderly, correct and flexible (re-combinable) musical auditory notions. Theoretical knowledge has to be used at the different stages of work on each specific piece, and its application is especially important at the time of initial acquaintance with the piece (the initial, most general analysis) and at the stage of detailed work - when learning the separate elements, 
memorizing by heart and establishing the pianist performer's conception of the play. At the last stage of work on mastering the piece - final, total inclusion, it is expected that it has been already analyzed and thought through in detail, but during the final review, the theoretical knowledge can be used in the context of revision, further clarification, comparison with earlier learnt works or with the other works from the concert or competition program.

Analyzing the work being learnt, with the goal of clarifying the performer's vision and memorization, consists of actualization of the musical auditory notions, their comparison (for example, in order to find what is common and what - different between the sections, for comparison of the culminations, needed to determine the main, most vivid culmination) and on this basis - further clarification of the dramaturgy of the play. It is appropriate the analysis to be done through reviewing the sheet music, without playing it for the moment, because during the process of performance on the piano, the attention of the instrumentalist is occupied with the specific practical tasks, while the analytical and generalizing view on the work requires to have a certain "distance" from the instrumental execution, from the motor realization of the piece. In this approach of acquiring musical auditory notions we count on the actualization of the notion of the previously heard music, supported and facilitated by referencing the sheet music, in the memory of the pianist, i.e. the analysis is in close relation with increasing the quality of the process of building and evoking the musical image, but is at its core also a clarification of this image.

Schematic study of the musical piece is a form of work, in which the play is not learnt in detail, since it is usually above the level of the instrumental skills of the student. The goal of this activity is to enrich the musical culture of the young pianist through playing a great number and more complicated works of the author being studied; to acquire impressions of the works (or a separate creative period) of the composer, whose opuses are unfamiliar to the student; getting acquainted with some musical phenomena (for example with depiction through music; a specific genre; an approach with a characteristic emotional impact; complex methro-rhythm and many others). Depending on the specific educative tasks, it is required to learn the piece in a slower than the original tempo, or to play it only several times, discussing the particularities, for which the work has been chosen as a musical example. In some cases, the practice can consist in performing only the themes, individual vivid excerpts, while the whole work is reviewed from the sheet music, from listening to the teacher's performance, or listening to recordings, followed by discussing the form, texture, dramaturgy, stylistic specifics of the work, the performing approaches. Of special value is the schematic acquaintance with numerous pieces by the composer, of whom there is a work of art, which is to be performed before of an audience. In this way, the young pianist finds themselves in the auditory atmosphere of the author being studied, in the composer's specific emotional scale, inherent to his imagery, his rhythm and intonation language. The natural result of this activity is the rapid creation and accumulation of musical auditory notions. This is of a great use for the pianist, especially if the following conditions have been fulfilled: the impressions that have arisen are verbally expressed, i.e. they have been consciously realized; the created notions are systemized under the skillful control of the pedagogue; conclusions have been formulated on their basis and all of this is used for the building of stable knowledge of the author's style; this knowledge the pianist will use in their future professional growth.

\section{Options of Practicing Supplementary Activities during the Piano Lesson}

In the various stages of professional growth of the instrumentalist, different options of enriching the auditory experience can be sought. Obviously, the piano literature dedicated to the youngest could not offer such a great variety, for example, of methro-rhythmic structures, as the more complex plays, being studied by older students. Besides, the child cannot be expected to assimilate complex information, or to learn to play a large volume of material in a short period of time. So, it will be suitable to use the following distribution of the tasks:

- The repertoire, designated for detailed mastering and performance is selected in such a way that learnt and consolidated with it, in addition to the instrument playing skills, will be the basic knowledge of note values, their correlation, ways of encoding, the more frequently used modal structures in the national and Western European musical traditions, i.e. so a stable basis of musical experience is being built. The plays, which have been studied in detail, will stay in the memory pool for a longer period of time, since participating in their memorization are several types of information - visual, containing the note text of the sheet and the needed for the performance keys; motor, including the motions of producing the tones; and auditory, representing the sound result. Since each piece, even the simplest one, possesses its own character, when it is discovered by the child, it adds a certain emotional coloring, which brings also emotional information into the memory pool. The fact that all types of memories are engaged in learning a single piece makes its memorization (respectively also the musical auditory notion about it) more reliable and lasting.

- During childhood, the musical auditory pool can be enhanced also by singing songs or by listening to the performances of the pedagogue and recordings of more complex works. The aim of this activity is to familiarize the child with the boundless world of expressive capabilities of the musical art, which 
motivates them to engage in extensive, diligent and regular activities with music, as well as to familiarize them with the varied piano literature for maximal enhancement of the musical auditory pool of the child. The accumulation of musical auditory notions is done as in the direction of consolidating the assimilated information in detail through similar musical examples, as well as for the demonstration of musical phenomena, different from the studied ones, for diversification of the pool of musical impressions of works that the child cannot yet master on the piano at this stage. Of course, most of the acquired in this way musical auditory notions for modal and methro-rhytmic phenomena are not required to be explained by introducing the specific terms, which identify them. It is important that they are perceived as a musical fact, that their character is discussed with the student, for the student to be able to connect the auditory impression with the emotional content; for them to be included in the pool of notions, even if not as the brightest comprising elements, so that they are not found unfamiliar, incomprehensible and dismissed by the student, when the suitable moment comes for them to perform similar music. When plays with musical phenomena, which the student has already encountered, are being listened to, the teacher has to ask for these phenomena to be identified. For the activation of the musical auditory notions, the pedagogue must direct the pupil to seek for similar phenomena among the plays, which they have studied, and to explain what is common between the new play, being taught at the moment, and the one that has been played at an earlier moment.

After their initial training has been completed, the young pianist has gathered a certain reserve of knowledge and skills, which allow them a more organized, focused and efficient self-reliant work. They are usually already more motivated to study piano and to succeed in learning the provisioned material and in performing it on stage, and also in their own presence before the audience. Their performances in concerts and competitions, up to the moment - episodic, almost exclusively in children forums - are now becoming more frequent. This brings to a new level also the responsibilities of the pedagogue to the pupil's professional growth, and from there - of the development of their musical abilities. The improved opportunities and motives for focused out-of-class work of the student can be used by the piano teacher also for applying another efficient approach for diversifying the rhythm and intonation language, with which the student is working - more frequent inclusion of supplementary activities like reading at first glance, playing by ear, schematic training, listening to recordings. The Russian piano pedagogue A. Shtapov [5] proposes several ways for freeing time for such activities in a piano lesson. Since a large portion of the skills for independent work have already been formed in the student, a habit has been created for auditory-visual-motor self-control, some instrumental skills have been learnt, the ability of reading independently through a new note text is present, the stereotype for everyday out-of-class activities on the piano has been established; so the work in the lesson, known as "training" [5] (the student practices under the extensive guidance of the teacher, who directs the goal and the approaches of reaching it, until a positive result is achieved within the very lesson) can be limited to the needed minimum, even though it cannot and should not be completely abandoned. This frees a certain amount of time, previously taken by the shared effort of overcoming the difficulties, teaching approaches of overcoming them and learning the basic habits of the pianist. Another way of finding time for supplementary activities in the lesson is alternating the musical works, which the pedagogue will hear being played from beginning to end. If in a single class session goals have been posed for the performance of several pieces, knowing the capabilities, the specific difficulties and individual pace of work of the student and depending on the timeframes, in which each piece should be prepared - in the consequent lesson, the teacher should decide which piece should be listened to entirely, of which piece only separate fragments can be reviewed and the performance of which piece can be postponed to the next lesson. If the tasks, on which the student has to work, are clear for them and they have experience in overcoming similar problems, but their solution requires more time, than the pieces, on which the student needs to solve this tasks, can be left without being performed during the lesson, or only separate episodes can be heard in order for the pedagogue to get an impression of whether this task have been clearly understood and at what stage of completion is the preparation of this works at the moment. Neither it is necessary to test the performance of the plays, which are at a stage of detailed work and to which tasks have been posed for the student, requiring work on specific fragments According to the provisions of the pedagogue, the revisal of the quality, which the student has reached in solving the tasks, can be limited to listening to those segments, which have created the most problems for the pupil in previous lessons; fragments, which the teacher supposes will have to be worked on during the lesson, or on which the next tasks needed to be set, additional clarification to be made, new approaches of work shown.

All supplementary activities in the lesson, regardless that some of them are aimed also at learning a specific type of skill, have one main aim, as well - to expand the musical culture of the student (i.e. enhancement of their musical auditory pool). Depending on the specific tasks in the lesson and on what is most crucial to be used with the individual student, the pieces, selected for the supplementary activities, can be by an author, who is being studied at the moment, or a composer, belonging to a different style, national school, time. 


\section{Conclusions}

1. Musical auditory notions are a musical ability, whose enhancement and perfection is extremely necessary for the piano performer.

2. Musical auditory notions can be improved in quantity and quality through professional training in piano.

3. For the development, clarification and enhancement of musical auditory notions, the main, as well as the supplementary activities in piano training can be used.

The thoughtful distribution of tasks within the piano lesson affects positively the successfulness of the student in specific forums, for which they are preparing, as well as in the long term - mastering their musical abilities, including, as well, the musical auditory notions.

\section{References}

[1] Golubovskaya, N. O. Of Musical Performance. L. 1985 (Голубовская, Н. О. О музыкальном исполнительстве. Л. 1985)

[2] Kogan, G. At the Gates of Mastery. The Work of the Pianist. Moscow, 1961 (Коган, Г. У врат мастерства. Работа пианиста. Москва, 1961)

[3] Kurteva, M. and J. Ganeva. Methodical Guide in Piano. Sofia 1984. (Куртева, М. и Дж. Ганева. Методическо ръководство по пиано. София, 1984.

[4] McKinnon, L. Playing by Heart. Leningrad, 1967 (Маккиннон, Л. Игра наизусть. Л. 1967)

[5] Shtapov, A. Piano Lesson in Musical Academy or School. Moscow, 2009 (Щапов, А. Фортепианный урок в музыкальной школе и училище. Москва, 2009 